ARTIFICIAL SATELLITES, Vol. 47, No. 3 - 2012

DOI: 10.2478/v10018-012-0018-6

\title{
STATISTICAL VIEW ON PHASE AND MAGNITUDE INFORMATION IN SIGNAL PROCESSING
}

\author{
Waldemar Popiński \\ Space Research Centre, PAS \\ e-mail: w.popinski@stat.gov.pl
}

\begin{abstract}
In this work the problem of reconstruction of an original complex-valued signal $o_{t}, t=0,1, \ldots, n-1$, from its Discrete Fourier Transform (DFT) spectrum corrupted by random fluctuations of magnitude and/or phase is investigated. It is assumed that the magnitude and/or phase of discrete spectrum values are distorted by realizations of uncorrelated random variables. The obtained results of analysis of signal reconstruction from such distorted DFT spectra concern derivation of the expected values and bounds on variances of the reconstructed signal at the observation moments. It is shown that the considered random distortions in general entail change in magnitude and/or phase of the reconstructed signal expected values, which together with imposed random deviations with finite variances can blur the similarity to the original signal. The effect of analogous random amplitude and/or phase distortions of a complex valued time domain signal on band pass filtration of distorted signal is also investigated.
\end{abstract}

Keywords: Discrete Fourier Transform, finite duration signal, discrete spectrum, magnitude/phase distortion, signal reconstruction, oscillation filtration.

\section{INTRODUCTION}

The well-known Discrete Fourier Transform (DFT) together with its inverse transform are widely used in signal and image analysis, representation and processing, involving tasks like filtering oscillations with specified frequency-bands from an observed signal (Gasquet and Witomski, 1999), (Koopmans, 1974) and image denoising or deblurring (Hansen et al., 2006), (Hoggar, 2006).

The DFT of a complex-valued time series $x_{t}, t=0,1, \ldots, n-1$, is computed as follows (Gasquet and Witomski, 1999):

$$
\tilde{x}_{\nu}=\sum_{t=0}^{n-1} x_{t} \exp (-i 2 \pi \nu t / n),
$$

for $\nu=0,1, \ldots, n-1$, and integer number $n>0$. The inverse transform is given by the formula (Gasquet and Witomski, 1999):

$$
x_{t}=\frac{1}{n} \sum_{\nu=0}^{n-1} \tilde{x}_{\nu} \exp (i 2 \pi t \nu / n) .
$$


Theoretical as well as numerical and statistical properties of the DFT are described in time series analysis textbooks (Blackledge, 2003), (Bloomfield, 2000), (Bremaud, 2002), (Brillinger, 1975), (Gasquet and Witomski, 1999), (Koopmans, 1974). Frequently, the appropriate Fast Fourier Transform (FFT) computer procedures are used to perform the relevant calculations (Cooley and Tukey, 1965), (Press et al., 1992), (Singleton, 1969).

It has been observed that the magnitudes and phases of the DFT spectra play different roles in one- and multidimensional signal reconstruction (Hayes et al., 1980). Namely, for preserving important features of a reconstructed signal the phases seem to be more relevant than magnitudes. This phenomenon of "phase importance" was described first by Oppenheim and Lim (1981), who performed numerical experiments to show the similarity between an original signal and reconstruction based on its DFT spectrum with unchanged phases. Ni and Huo (2007) presented certain explanation of this phenomenon from the perspective of statistical interpretation of phase information in signal reconstruction. The aim of this work is to pursue the same subject further and show results which significantly complete their conclusions and present facts which were not remarked in their work. The presented investigations are based on the idea of introducing random distortions of an original signal DFT spectrum magnitudes and/or phases, and examining the effects such a modification has on the reconstructed signal.

The assumed concept of time domain signal or its spectrum random distortion modeling is described in section 2. The theoretical results related to the reconstruction of a signal from its distorted DFT spectrum are presented both in the case of a noiseless signal (section 3) and in the case of signal observations corrupted by uncorrelated random errors (section 4). In section 5 the effects that considered random amplitude and/or phase distortions of a time domain signal produce when distorted signal is filtered using the Fourier Transform Band Pass Filter (FTBPF) technique are examined.

\section{PHASE AND MAGNITUDE RANDOM DISTORTION MODELING}

Let us consider finite duration time series of the complex-valued signal measurements $o_{t}$ at discrete equidistant time moments $t=0,1, \ldots, n-1$, and its discrete spectrum $\tilde{o}_{\nu}, \nu=$ $0,1, \ldots, n-1$, computed according to (1). Now, let the magnitude and phase of the signal discrete spectrum values be distorted by random variables $a_{\nu}$ and $\phi_{\nu}$, respectively, according to the mathematical model

$$
\tilde{v}_{\nu}=a_{\nu} \exp \left(i \phi_{\nu}\right) \tilde{o}_{\nu}=u_{\nu} \tilde{o}_{\nu}
$$

where $u_{\nu}=a_{\nu} \exp \left(i \phi_{\nu}\right)$. Hence, we can consider separately random magnitude or phase distortions, as well as joint magnitude and phase spectrum distortion.

Let us assume that $\phi_{\nu}$ and $a_{\nu}, \nu=0,1, \ldots, n-1$, are realizations of independent and identically distributed random variables, and magnitude distortions are independent of phase distortions. We further assume that the random magnitude fluctuations $a_{\nu}$ are nonnegative and the random phase fluctuations $\phi_{\nu}$ take their values in the interval $[0,2 \pi)$. Obviously, $E_{\phi} \exp \left(i \phi_{\nu}\right)=\chi_{\phi}(1)$, where $\chi_{\phi}(t), t \in \mathbb{R}$, denotes the characteristic function of the phase distortions, and consequently

$$
\sigma_{\phi}^{2}=E_{\phi}\left|\exp \left(i \phi_{\nu}\right)-\chi_{\phi}(1)\right|^{2}=E_{\phi}\left|\exp \left(i \phi_{\nu}\right)\right|^{2}-\left|\chi_{\phi}(1)\right|^{2}=1-\left|\chi_{\phi}(1)\right|^{2} \leq 1 .
$$

For example, let the distribution of $\phi_{\nu}$ be uniform on the interval $(-\Phi, \Phi)$, i.e. $\phi_{\nu} \sim$ 
$U(-\Phi, \Phi)$, where $0<\Phi \leq \pi$, which gives immediately

$$
m(\Phi)=E_{\phi} \exp \left(i \phi_{\nu}\right)=\frac{1}{2 \Phi} \int_{-\Phi}^{\Phi} \exp (i x) d x=\frac{\sin (\Phi)}{\Phi},
$$

so as one can clearly see $0 \leq m(\Phi)<1$ and $m(\Phi)=0$ only for $\Phi=\pi$.

According to the assumptions on the distribution of the real-valued random variables $a_{\nu}$ we have $m_{a}=E_{a} a_{\nu} \geq 0$ and we further assume that $\sigma_{a}^{2}=E_{a}\left|a_{\nu}-E_{a} a_{\nu}\right|^{2}<\infty$ (if $E_{a} a_{\nu}=1$ and $\sigma_{a}=0$ there is only phase distortion of the signal spectrum). For instance, if $a_{\nu}$ have Rayleigh distribution on the interval $[0, \infty)$, with density $p_{\alpha}(t)=\frac{t}{\alpha^{2}} \exp \left(-t^{2} / 2 \alpha^{2}\right)$, where $\alpha>0$, then $m_{a}(\alpha)=\alpha \sqrt{\pi / 2}, \sigma_{a}^{2}(\alpha)=\alpha^{2}(4-\pi) / 2$ (Johnson et al., 1994).

The above assumptions further imply for $u_{\nu}=a_{\nu} \exp \left(i \phi_{\nu}\right)$,

$$
\begin{gathered}
m_{u}=E_{a} E_{\phi} a_{\nu} \exp \left(i \phi_{\nu}\right)=E_{a} a_{\nu} E_{\phi} \exp \left(i \phi_{\nu}\right)=m_{a} \chi_{\phi}(1) \\
\sigma_{u}^{2}=E_{\phi} E_{a}\left|a_{\nu} \exp \left(i \phi_{\nu}\right)-m_{a} \exp \left(i \phi_{\nu}\right)+m_{a} \exp \left(i \phi_{\nu}\right)-m_{a} \chi_{\phi}(1)\right|^{2} \\
=E_{a}\left|a_{\nu}-m_{a}\right|^{2} E_{\phi}\left|\exp \left(i \phi_{\nu}\right)\right|^{2}+m_{a}^{2} E_{\phi}\left|\exp \left(i \phi_{\nu}\right)-\chi_{\phi}(1)\right|^{2} \\
=\sigma_{a}^{2}+m_{a}^{2}\left(1-\left|\chi_{\phi}(1)\right|^{2}\right),
\end{gathered}
$$

and obviously we have $\sigma_{a}^{2} \leq \sigma_{u}^{2} \leq \sigma_{a}^{2}+m_{a}^{2}$.

Our aim is to characterize the signal reconstructed from distorted DFT spectrum of an original signal. Hence, according to our model (3) we have to analyze the inverse DFT of the discrete spectrum of the form $\tilde{v}_{\nu}=u_{\nu} \tilde{O}_{\nu}, \nu=0,1, \ldots, n-1$, where distortions are represented by realizations of complex-valued random variables $u_{\nu}$. Indeed, the cases of $u_{\nu}=\exp \left(i \phi_{\nu}\right), u_{\nu}=a_{\nu} \exp \left(i \phi_{\nu}\right)$ and $u_{\nu}=a_{\nu}$ correspond to phase, phase and magnitude, and magnitude distortions, respectively.

In Lemma 2.1 the DFT of a distorted time domain signal and the inverse DFT of distorted spectrum are characterized, when distortions are represented by a finite sample od uncorrelated random variables with identical first and second moments.

LEMMA 2.1. Let $x_{t}, t=0,1, \ldots, n-1$, be a finite sequence of complex numbers and assume that the complex-valued random variables $Z_{t}, t=0,1, \ldots, n-1$, are uncorrelated, and their mean values and variances satisfy the conditions $E_{z} Z_{t}=m_{z}$ and $E_{z}\left|Z_{t}-m_{z}\right|^{2}=$ $\sigma_{z}^{2}<\infty$. If we define $z_{t}=Z_{t} x_{t}$ or $\tilde{z}_{\nu}=Z_{\nu} \tilde{x}_{\nu}, \nu=0,1, \ldots, n-1$, then

or

$$
E_{z} \tilde{z}_{\nu}=m_{z} \tilde{x}_{\nu} \quad, \quad E_{z}\left|\tilde{z}_{\nu}-E_{z} \tilde{z}_{\nu}\right|^{2}=\sigma_{z}^{2} \sum_{t=0}^{n-1}\left|x_{t}\right|^{2} \quad \text { for } \quad \nu=0,1, \ldots, n-1,
$$

$$
E_{z} z_{t}=m_{z} x_{t} \quad, \quad E_{z}\left|z_{t}-E_{z} z_{t}\right|^{2}=\sigma_{z}^{2} \frac{1}{n^{2}} \sum_{\nu=0}^{n-1}\left|\tilde{x}_{\nu}\right|^{2} \quad \text { for } \quad t=0,1, \ldots, n-1,
$$

respectively.

Proof. In the case where $z_{t}=Z_{t} x_{t}, t=0,1, \ldots, n-1$, the assumptions of the lemma and definition (1) yield for $\nu=0,1, \ldots, n-1$,

$$
E_{z} \tilde{z}_{\nu}=m_{z} \sum_{t=0}^{n-1} x_{t} \exp (-i 2 \pi \nu t / n)=m_{z} \tilde{x}_{\nu}
$$

Further, the assumed zero correlation of the random variables $Z_{t}, t=0,1, \ldots, n-1$, implies 


$$
\begin{gathered}
E_{z}\left|\tilde{z}_{\nu}-E_{z} \tilde{z}_{\nu}\right|^{2}=E_{z} \sum_{t=0}^{n-1}\left(Z_{t}-E_{z} Z_{t}\right) x_{t} \exp (-i 2 \pi \nu t / n) \sum_{s=0}^{n-1}\left(\bar{Z}_{s}-E_{z} \bar{Z}_{s}\right) \bar{x}_{t} \exp (i 2 \pi \nu s / n) \\
=\sum_{t=0}^{n-1} \sum_{s=0}^{n-1} E_{z}\left(Z_{t}-E_{z} Z_{t}\right)\left(\bar{Z}_{s}-E_{z} \bar{Z}_{s}\right) x_{t} \bar{x}_{s} \exp (i 2 \pi \nu(s-t) / n)=\sigma_{z}^{2} \sum_{t=0}^{n-1}\left|x_{t}\right|^{2} .
\end{gathered}
$$

The proof in the case where $\tilde{z}_{\nu}=Z_{\nu} \tilde{x}_{\nu}$ is analogous, but we use the definition (2) of the inverse transform.

\section{RECONSTRUCTED SIGNAL PROPERTIES}

Lemma 2.1 allows us to characterize the inverse DFT of spectra corresponding to the distortion models considered. Namely, we can derive formulae for the mean values and variances of the random variables $v_{t}, t=0,1, \ldots, n-1$, representing signals reconstructed from distorted spectra defined in (3). The following corollary concerns the case of signals with bounded spectra $\tilde{o}_{\nu}, \nu=0,1, \ldots, n-1$.

COROLLARY 3.1. If $\left|\tilde{o}_{\nu}\right| \leq B<\infty, \nu=0,1, \ldots, n-1$, and the assumptions of Lemma 2.1 hold, then the inverse DFT of the discrete spectrum $\tilde{r}_{\nu}=Z_{\nu} \tilde{o}_{\nu}, \nu=0,1, \ldots, n-1$, satisfies for $t=0,1, \ldots, n-1$,

$$
E_{z}\left|r_{t}-E_{z} r_{t}\right|^{2} \leq \frac{\sigma_{z}^{2} B^{2}}{n}
$$

As Lemma 2.1 shows DFT spectrum distortion according to our model can make both the magnitudes and phases of the reconstructed signal mean values $E_{z} r_{t}$ differ from the magnitudes and phases of the original signal $o_{t}, t=0,1, \ldots, n-1$. The magnitudes of all the reconstructed signal mean values are multiplied by the constant factor $\left|m_{z}\right|$, and their phases are changed by the constant additive term $\phi_{z}$, where $m_{z}=\left|m_{z}\right| \exp \left(i \phi_{z}\right)$. Phase distortion of the reconstructed signal mean values does not occur if $m_{z}$ is real-valued, like in the example described in section 2. Such changes of the magnitudes and phases of the reconstructed signal values are clealy of non-random character. However, there are also random effects of signal reconstruction from distorted spectrum, which are characterized by the variances of the random variables $r_{t}, t=0,1, \ldots, n-1$. If the spectrum distorting sequence $Z_{\nu}, \nu=0,1, \ldots, n-1$, comprises realizations of uncorrelated random variables with identical mean values and variances, then according to Corollary 3.1 the variances $\operatorname{Var}_{z}\left(r_{t}\right), t=0,1, \ldots, n-1$, decrease uniformly to zero as $n \rightarrow \infty$, whenever the original signal spectrum $\tilde{o}_{\nu}, \nu=0,1, \ldots, n-1$, is bounded. In consequence, in the case considered, the influence of the resulting random signal deviations $r_{t}-E_{z} r_{t}, t=0,1, \ldots, n-1$, on the reconstructed signal diminishes asymptotically as $n \rightarrow \infty$. This means that they will not make the signal values reconstructed from the distorted spectrum differ significantly from their mean values.

Hence, any peaks present in the original signal magnitudes $\left|o_{t}\right|$ may be less distinguishable in the reconstructed signal magnitudes $\left|r_{t}\right|$, especially when $\left|m_{z}\right|<1$. In the extreme case of $m_{z}=0$ we have $E_{z} r_{t}=0$, and then the reconstructed signal will have purely stochastic character, so its similarity to the original signal will be completely blurred.

Using the well-known property of the DFT (Gasquet and Witomski, 1999)

$$
\sum_{t=0}^{n-1}\left|x_{t}\right|^{2}=\frac{1}{n} \sum_{\nu=0}^{n-1}\left|\tilde{x}_{\nu}\right|^{2}
$$


we can also derive a formula for the expected value of the squared euclidean distance between the vectors $\left(o_{0}, o_{1}, \ldots, o_{n-1}\right)$ and $\left(r_{0}, r_{1}, \ldots, r_{n-1}\right)$, representing the original and reconstructed signal, respectively. Indeed, applying the above formula to the differences $r_{t}-o_{t}$ and putting $\tilde{r}_{\nu}=Z_{\nu} \tilde{o}_{\nu}, \nu=0,1, \ldots, n-1$, we obtain the equality

$E_{z} \sum_{t=0}^{n-1}\left|r_{t}-o_{t}\right|^{2}=\frac{1}{n} \sum_{\nu=0}^{n-1} E_{z}\left|\tilde{r}_{\nu}-\tilde{o}_{\nu}\right|^{2}=\frac{1}{n} \sum_{\nu=0}^{n-1}\left|\tilde{o}_{\nu}\right|^{2} E_{z}\left|Z_{\nu}-1\right|^{2}=\left(\sigma_{z}^{2}+\left|m_{z}-1\right|^{2}\right) \frac{1}{n} \sum_{\nu=0}^{n-1}\left|\tilde{o}_{\nu}\right|^{2}$

from which it follows easily that for a signal with bounded spectrum $\left|\tilde{o}_{\nu}\right| \leq B<\infty, \nu=$ $0,1, \ldots, n-1$, the computed squared euclidean distance mean value has an upper bound $\left(\sigma_{z}^{2}+\left|m_{z}-1\right|^{2}\right) B^{2}$.

Coming back to the spectrum magnitude and/or phase distortion model (3) considered in the section 2 , there is one general observation worth mentioning that follows from the above characterization of reconstructed signals. Namely, for any distribution of the spectrum magnitude distortions $a_{\nu}, \nu=0,1, \ldots, n-1$, the mean values of the signal reconstructed from the magnitude distorted original signal spectrum will represent the scaled version of the original signal with the scaling factor $m_{a}=E_{a} a_{\nu}$, i.e. $E_{a} v_{t}=$ $m_{a} o_{t}, t=0,1, \ldots, n-1$ (except for the two extreme cases where $m_{a}=\infty$ or $m_{a}=0$ ). On the other hand, if only spectrum phase distortions $\phi_{\nu}$ are present in the model (3), then we will have the relation $E_{\phi} v_{t}=\chi_{\phi}(1) o_{t}, t=0,1, \ldots, n-1$. Consequently, since $\chi_{\phi}(1)=\left|\chi_{\phi}(1)\right| \exp (i \Phi)$, where $0 \leq \Phi<2 \pi$, can be complex-valued, this relation implies $\left|E_{\phi} v_{t}\right|=\left|\chi_{\phi}(1)\right|\left|o_{t}\right| \leq\left|o_{t}\right|$, but now the mean values $E_{\phi} v_{t}$ can be also rotated in the complex plane with respect to $o_{t}$, if $\Phi \neq 0$. Moreover, there exist nontrivial distributions of phase distortions for which $\chi_{\phi}(1)=0$ (e.g. $\phi_{\nu} \sim U((-\pi, \pi)$ ), and for such distributions similarity between the original signal and the signal reconstructed from its phase distorted spectrum can be completely blurred. Generally, if a random variable $X$ has characteristic function which satisfies $\chi_{X}\left(t_{0}\right)=0$ for some $t_{0} \in \mathbb{R}$, then the characteristic function of the random variable $Y=t_{0} X$ satisfies $\chi_{Y}(1)=0$, since $\chi_{Y}(t)=E \exp (i t Y)$ for $t \in \mathbb{R}$.

The observed properties shed some light on the difference between spectrum magnitude and phase information in signal reconstruction.

\section{RECONSTRUCTION OF NOISY SIGNALS}

Assume now that the time series of the original signal values is corrupted by random observation errors, according to the model

$$
y_{t}=o_{t}+\eta_{t}, \quad t=0,1, \ldots, n-1,
$$

where $\eta_{t}$ are realizations of uncorrelated complex-valued random variables having zero mean $E_{\eta} \eta_{t}=0$ and finite second moment $\sigma_{\eta}^{2}=E_{\eta}\left|\eta_{t}\right|^{2}<\infty$.

Let us see what happens if the corrupted signal spectrum values are submitted to random distortions of the same kind as considered above, i.e. $\tilde{v}_{\nu}=u_{\nu} \tilde{y}_{\nu}, \nu=0,1, \ldots, n-1$. In the sequel we assume that the observation errors $\eta_{t}$ are independent of the random variables $u_{\nu}$ forming the distorting sequence. We can prove the following lemma.

LEMMA 4.1. Let $y_{t}, t=0,1, \ldots, n-1$, be a finite sequence of observations according to model (4) and assume that the complex-valued random variables $Z_{t}, t=0,1, \ldots, n-1$, are uncorrelated, and their mean values and variances satisfy the conditions $E_{z} Z_{t}=m_{z}$ and $E_{z}\left|Z_{t}-m_{z}\right|^{2}=\sigma_{z}^{2}<\infty$. If we define $s_{t}=Z_{t} y_{t}$, or $\tilde{s}_{\nu}=Z_{\nu} \tilde{y}_{\nu}, \nu=0,1, \ldots, n-1$, then 


$$
E_{\eta} E_{z} \tilde{s}_{\nu}=m_{z} \tilde{o}_{\nu} \quad \text { and } \quad E_{\eta} E_{z}\left|\tilde{s}_{\nu}-E_{\eta} E_{z} \tilde{s}_{\nu}\right|^{2}=\sigma_{z}^{2} \sum_{t=0}^{n-1}\left|o_{t}\right|^{2}+n\left(\sigma_{z}^{2}+\left|m_{z}\right|^{2}\right) \sigma_{\eta}^{2}
$$

for $\nu=0,1, \ldots, n-1$, or

$$
E_{\eta} E_{z} s_{t}=m_{z} o_{t} \quad \text { and } \quad E_{\eta} E_{z}\left|s_{t}-E_{\eta} E_{z} s_{t}\right|^{2}=\frac{\sigma_{z}^{2}}{n^{2}} \sum_{\nu=0}^{n-1}\left|\tilde{o}_{\nu}\right|^{2}+\left(\sigma_{z}^{2}+\left|m_{z}\right|^{2}\right) \sigma_{\eta}^{2}
$$

for $t=0,1, \ldots, n-1$, respectively.

Proof. In the case where $s_{t}=Z_{t} y_{t}, t=0,1, \ldots, n-1$, since the assertions of Lemma 2.1 hold, it assures that $E_{z} \tilde{s}_{\nu}=m_{z} \tilde{y}_{\nu}$ and by (4) we have $E_{\eta} E_{z} \tilde{s}_{\nu}=m_{z} E_{\eta}\left(\tilde{o}_{\nu}+\tilde{\eta}_{\nu}\right)=m_{z} \tilde{o}_{\nu}$ for $\nu=0,1, \ldots, n-1$. Furthermore, by Lemma 2.1

$$
E_{z}\left|\tilde{s}_{\nu}-E_{z} \tilde{s}_{\nu}\right|^{2}=\sigma_{z}^{2} \sum_{t=0}^{n-1}\left|y_{t}\right|^{2}
$$

and simple calculation shows that

$$
\begin{aligned}
E_{z}\left|\tilde{s}_{\nu}-E_{\eta} E_{z} \tilde{s}_{\nu}\right|^{2}= & E_{z}\left|\tilde{s}_{\nu}-E_{z} \tilde{s}_{\nu}+E_{z} \tilde{s}_{\nu}-E_{\eta} E_{z} \tilde{s}_{\nu}\right|^{2} \\
& =E_{z}\left|\tilde{s}_{\nu}-E_{z} \tilde{s}_{\nu}\right|^{2}+\left|E_{z} \tilde{s}_{\nu}-E_{\eta} E_{z} \tilde{s}_{\nu}\right|^{2}=E_{z}\left|\tilde{s}_{\nu}-E_{z} \tilde{s}_{\nu}\right|^{2}+\left|m_{z}\left(\tilde{y}_{\nu}-\tilde{o}_{\nu}\right)\right|^{2} .
\end{aligned}
$$

Hence, the above equalities together with (4) imply

$E_{\eta} E_{z}\left|\tilde{s}_{\nu}-E_{\eta} E_{z} \tilde{s}_{\nu}\right|^{2}=\sigma_{z}^{2} \sum_{t=0}^{n-1} E_{\eta}\left|o_{t}+\eta_{t}\right|^{2}+E_{\eta}\left|m_{z} \tilde{\eta}_{\nu}\right|^{2}=\sigma_{z}^{2} \sum_{t=0}^{n-1}\left[\left|o_{t}\right|^{2}+E_{\eta}\left|\eta_{t}\right|^{2}\right]+n\left|m_{z}\right|^{2} \sigma_{\eta}^{2}$

which completes the proof. The proof in the case where $\tilde{s}_{\nu}=Z_{\nu} \tilde{y}_{\nu}$ is analogous, except that we use the second assertion of Lemma 2.1.

On the basis of Lemma 4.1 we can easily deduce corollary analogous to 3.1 for the case of signals with bounded spectrum $\tilde{o}_{\nu}, \nu=0,1, \ldots, n-1$. Thus, we see that presence of the considered zero-mean uncorrelated errors, corrupting the original signal values, does not change the character of the signal reconstructed from the distorted DFT spectrum. Indeed, the formulae for the reconstructed signal mean values remain the same as in the case of errorless signal, and the reconstructed signal variances in Lemma 4.1 differ from those of Lemma 2.1 by the relevant additive terms which occur because of non-zero second moment of the observation errors. However, presence of these variance components also implies that the reconstructed signal variances do not converge uniformly to zero as $n \rightarrow \infty$ for signals with bounded spectrum.

\section{FILTRATION OF RANDOMLY MODULATED SIGNALS}

Now, we shall examine the effects that random amplitude and/or phase modulation of the deterministic signal $o_{t}, t=0,1, \ldots, n-1$, according to the model analogous to $(3)$,

$$
v_{t}=a_{t} \exp \left(i \phi_{t}\right) o_{t}=u_{t} o_{t}
$$

where $u_{t}=a_{t} \exp \left(i \phi_{t}\right)$, can produce in the case when the modulated signal $v_{t}$ is filtered using the well-known Fourier Transform Band-Pass Filter (Koopmans, 1974),(Popiński, 2008). Let the assumptions on idependence and distribution of the random magnitude $a_{t}$ and phase $\phi_{t}$ distortions be the same as formulated for model (3). The original deterministic signal can represent for example a complex monochromatic oscillation with constant frequency, amplitude and phase, i.e. $o_{t}=A \exp (i \omega t+i \phi)$, where $\omega \neq 0, A>0, \phi \in[0,2 \pi)$. 
In its discrete version the output of the FTBPF applied on the finite duration complexvalued time series $x_{t}, t=0,1, \ldots, n-1$, is defined for even $n$ as (Koopmans, 1974):

$$
\hat{x}_{t}=\frac{1}{n} \sum_{\nu=-n / 2+1}^{n / 2} A(2 \pi \nu / n) \tilde{x}_{\nu} \exp (i 2 \pi t \nu / n),
$$

where $0 \leq A(\omega) \leq 1$ is the transmittance function of the filter, different from zero in a neighbourhood of some chosen frequency $-\pi \leq \omega_{0} \leq \pi$, i.e. $A(\omega)=0$ for $\left|\omega-\omega_{0}\right| \geq 2 \pi \lambda$ and given pass-band width parameter $\lambda>0$. In the definition (6) the range of summation index is $-n / 2+1 \leq \nu \leq n / 2$ but periodicity of the DFT assures that $\tilde{x}_{-\nu}=\tilde{x}_{n-\nu}, \nu=$ $1, \ldots, n / 2-1$, so it is enough to compute the values $\tilde{x}_{\nu}, \nu=0,1, \ldots, n-1$.

Since the assumptions of Lemma 2.1 hold, equality (5) implies

$$
\tilde{v}_{\nu}=E_{u} \tilde{v}_{\nu}+\left(\tilde{v}_{\nu}-E_{u} \tilde{v}_{\nu}\right)=m_{u} \tilde{o}_{\nu}+\left(\tilde{v}_{\nu}-E_{u} \tilde{v}_{\nu}\right)
$$

for $\nu=0,1, \ldots, n-1$, where $m_{u}=E_{u} u_{t}$, and $E_{u}\left|\tilde{v}_{\nu}-E_{u} \tilde{v}_{\nu}\right|^{2}=\sigma_{u}^{2} \sum_{t=0}^{n-1}\left|o_{t}\right|^{2}$ with $\sigma_{u}^{2}=E_{u}\left|u_{t}-m_{u}\right|^{2}$. Consequently, band-pass filtration according to (6), applied on the modulated signal, yields immediately

$$
\hat{v}_{t}=m_{u} \hat{o}_{t}+\frac{1}{n} \sum_{\nu=-n / 2+1}^{n / 2} A(2 \pi \nu / n)\left(\tilde{v}_{\nu}-E_{u} \tilde{v}_{\nu}\right) \exp (i 2 \pi t \nu / n)
$$

for $t=0,1, \ldots, n-1$. Furthermore, $E_{u} \hat{v}_{t}=m_{u} \hat{o}_{t}$ and since $A(\omega)=0$ for $\left|\omega-\omega_{0}\right| \geq 2 \pi \lambda$ we have

$$
E_{u}\left|\hat{v}_{t}-m_{u} \hat{o}_{t}\right|^{2}=\left|\frac{1}{n} \sum_{\nu=\nu_{1}+1}^{\nu_{2}} A(2 \pi \nu / n)\left(\tilde{v}_{\nu}-E_{u} \tilde{v}_{\nu}\right) \exp (i 2 \pi t \nu / n)\right|^{2}
$$

for $t=0,1, \ldots, n-1$, where integers $-n / 2 \leq \nu_{1}<\nu_{2} \leq n / 2$ are chosen so that $A(2 \pi \nu / n) \neq 0$ for $\nu_{1}+1 \leq \nu \leq \nu_{2}$. The formula for $E_{u}\left|\tilde{v}_{\nu}-E_{u} \tilde{v}_{\nu}\right|^{2}$ together with the Schwartz inequality gives

$$
E_{u}\left|\hat{v}_{t}-m_{u} \hat{o}_{t}\right|^{2} \leq \frac{\left(\nu_{2}-\nu_{1}\right)}{n} \sigma_{u}^{2} \sum_{t=0}^{n-1}\left|o_{t}\right|^{2} \frac{1}{n} \sum_{\nu=\nu_{1}+1}^{\nu_{2}}|A(2 \pi \nu / n)|^{2} \leq \frac{\left(\nu_{2}-\nu_{1}\right)^{2}}{n^{2}} \sigma_{u}^{2} \sum_{t=0}^{n-1}\left|o_{t}\right|^{2}
$$

for $t=0,1, \ldots, n-1$. Hence, if the original signal is bounded, i.e. $\left|o_{t}\right| \leq B<\infty, t=$ $0,1, \ldots, n-1$, then we obtain the following upper for the variance of the filtered modulated signal

$$
E_{u}\left|\hat{v}_{t}-m_{u} \hat{o}_{t}\right|^{2} \leq \frac{\left(\nu_{2}-\nu_{1}\right)^{2}}{n} \sigma_{u}^{2} B^{2} .
$$

If we assume that the transition band $\left(2 \pi \nu_{1}(n) / n, 2 \pi \nu_{2}(n) / n\right]$ is concentrated around some fixed frequency $\omega_{0}$ and gets narrower and narrower as the data number $n$ grows, so that the difference $\nu_{2}(n)-\nu_{1}(n)$ remains constant, then from the above inequality (7) it follows immediately that the series $\hat{v}_{t}$ represents consistent in the mean sense estimates of $E_{u} \hat{v}_{t}=m_{u} \hat{o}_{t}$, i.e. the scaled oscillation series filtered from the original signal data. Thus, we can formulate the following corollary.

COROLLARY 5.1. If $\left|o_{t}\right| \leq B<\infty$ and $v_{t}=u_{t} o_{t}, t=0,1, \ldots, n-1$, then the time series $\hat{v}_{t}$ filtered by the FTBPF satisfies 
where $A(2 \pi \nu / n) \neq 0$ for $\nu_{1}+1 \leq \nu \leq \nu_{2}$.

$$
E_{u}\left|\hat{v}_{t}-E_{u} v_{t}\right|^{2} \leq \frac{\left(\nu_{2}-\nu_{1}\right)^{2}}{n} \sigma_{u}^{2} B^{2}
$$

The observations of section 3 are also valid in the considered case of FTBP filtration of a randomly distorted time domain signal. Namely, random amplitude and/or phase modulation of a signal according to our simple distortion model can change both amplitude and phase of the filtered oscillation mean values $E_{u} \hat{v}_{t}=m_{u} \hat{o}_{t}, t=0,1, \ldots, n-1$, which represent the oscillation filtered from the original signal, with amplitudes scaled by the constant factor $\left|m_{u}\right|$, and phases changed by the additive term $\phi_{u}$, where $m_{u}=\left|m_{u}\right| \exp \left(i \phi_{u}\right)$.

In view of Lemma 4.1 corollary analogous to 5.1 remains valid also in the case when instead of errorless signal values we use their observations corrupted by uncorrelated errors according to model (4)(Popiński, 1997).

\section{CONCLUSIONS}

The properties of the signal reconstruction from its distorted DFT spectrum, examined in this work, are helpful for understanding the possible changes such a reconstruction undergoes in the case of random magnitude and/or phase distortion of the original signal spectrum. Our distortion model includes fluctuations of stochastic nature in the magnitudes and/or phases of the original signal spectrum values. For deterministic signals it is proved that occurrence of random phase distortions of the signal spectrum can completely change the character of the reconstructed signal. Namely, the signal reconstructed from its phase distorted spectrum may show purely stochastic character quite different from the original deterministic signal. On the other hand for original signals with bounded spectrum it is also shown that in certain cases the signal reconstructed from the distorted spectrum can still resemble the original signal, although small peaks can be significantly smoothed. Similar conclusions are deduced also in the case of a deterministic signal which is corrupted at the moments of observation by uncorrelated random errors with zero mean and finite variance.

On the basis of the obtained results certain difference between the magnitude and phase information contained in the signal spectrum is indicated, which confirms observations of other authors that similarity of the signal reconstructed from the spectrum to the original signal is more sensitive to phase distortions than to magnitude distortions.

Since the DFT is linear, the obtained results also help to understand the influence particular signal component spectrum distortion can have on reconstruction of a signal which is a sum of several components (e.g. monochromatic oscillations).

Here we confined our investigation to the case of one-dimensional signals but its extension to the multidimensional case is straightforward in view of the tensor-product structure of the multidimensional DFT.

Let us remark that similar analysis can be also applied in the case of additive magnitude distortions, i.e if we put $a_{\nu}=1+b_{\nu}, \nu=0,1, \ldots, n-1$, in the model (3), where the real-valued random variables $b_{\nu}$ are assumed to be independent, identically distributed, and satisfy the conditions $E_{b} b_{\nu}=m_{b}$ and $E_{b}\left(b_{\nu}-E_{b} b_{\nu}\right)^{2}<\infty$.

The concept of phase randomization used for obtaining multivariate surrogate time series (Mammen and Sandi, 2008), (Schreiber and Schmitz, 2000) with distribution similar to an observed series is also related to the subject considered here. Moreover, similar kind of random phase distortions of a time series DFT spectrum as the one considered here is applied in the Fourier Bootstrap Method (Brown and Kulperger, 1997). 
In accordance with our assertions concerning the influence of random amplitude and/or phase modulation of a time domain signal on the FTBP filtration results, mean values of oscillations filtered from such a modulated signal are similar to oscillations filtered from the original signal, but their amplitudes are scaled by a constant factor and their phases are changed by a constant offset.

Acknowledgments. This research work was supported by the Polish National Science Centre through the grant No. N 526157040 carried out at the Space Research Centre of Polish Academy of Sciences.

\section{REFERENCES}

Blackledge J. M. (2003). Digital Signal Processing, Horwood Publishing, Chichester, West Sussex, England.

Bloomfield P. (2000). Fourier Analysis of Time Series: An Introduction, Wiley, New York.

Braun W. J., Kulperger R. J. (1997). Properties of a Fourier Bootstrap Method for Time Series, Communications in Statistics - Theory and Methods, 26(6), 1329-1335.

Bremaud P. (2002). Mathematical Principles of Signal Processing: Fourier and Wavelet Analysis, Springer Verlag Inc., New York.

Brillinger D. R. (1975). Time Series - Data Analysis and Theory, Holt, Rinehart and Winston Inc., New York.

Cooley J. W. and Tukey J. W. (1965). An Algorithm for the Machine Calculation of Complex Fourier Series, Mathematics of Computation, 19, 297-301.

Gasquet C., Witomski P. (1999). Fourier Analysis and Applications - Filtering, Numerical Computation, Wavelets, Springer Verlag Inc., New York.

Hansen P. Ch., Nagy J. G., O’Leary D. P. (2006). Deblurring Images, Matrices, Spectra and Filtering, SIAM, Philadelphia.

Hayes M., Lim J., Oppenheim A. (1980). Signal Reconstruction from Phase or Magnitude, IEEE Transactions on Acoustics Speech and Signal Processing, ASSP-28(6), pp. 672680.

Hoggar S. D. (2006). Mathematics of Digital Images - Creation, Compression, Restoration, Recognition, Cambridge University Press, Cambridge.

Johnson N.L., Kotz S., Balakrishnan N. (1994). Continuous Univariate Distributions, Vol. 1-2, John Wiley \& Sons, New York.

Koopmans L. H. (1974). The Spectral Analysis of Time Series, Academic Press, New York.

Mammen E. and Nandi S. (2008). Some Theoretical Properties of Phase-Randomized Multivariate Surrogates, Statistics, 42(3), 195-205.

Ni X. and Huo X. (2007). Statistical Interpretation of the Importance of Phase Information in Signal and Image Reconstruction, Statistics and Probability Letters, 77(4), 447-454.

Oppenheim A. V., Lim J. S. (1981). The Importance of Phase in Signals, Proceedings of the IEEE, 69(5), 529-541.

Popiński W. (1997). On Consistency of Discrete Fourier Analysis of Noisy Time Series, Artificial Satellites - Journal of Planetary Geodesy, 32(3), 131-142. 
Popiński W. (2008). Insight into the Fourier Transform Band Pass Filtering Technique, Artificial Satellites - Journal of Planetary Geodesy, 43(4), 129-141.

Popiński W. (2010). On Discrete Fourier Spectrum of Randomly Modulated Signals, Artificial Satellites - Journal of Planetary Geodesy, 45(3), 143-152.

Press W. H., Flannery B. P., Teukolsky S. A., and Vetterling W. T. (1992). Numerical Recipes - The Art of Scientific Computing, Cambridge University Press, Cambridge.

Schreiber T., Schmitz A. (2000). Surrogate Time Series, Physica D, 142(3-4), 346-382.

Singleton R. C. (1969). An Algorithm for Computing the Mixed Radix Fast Fourier Transform, IEEE Transactiions on Audio and Electroacoustics, AU-17(2), 93-103.

Received: 2012-06-29,

Reviewed: 2012-07-18, by W. Kosek,

Accepted: 2012-08-01. 